\title{
Modeling and Simulation Analysis of Steering Stability Based on Vehicle Motion Simulator
}

\author{
Lijun Qi, Wen Xu, Feng Zhang \\ Aviation University of Air Force, Changchun, 130022, China
}

Keywords:.driving simulation, vehicle dynamics model, dynamics model simulation.

\begin{abstract}
In this paper, several steering inputs affecting vehicle motion status is taken fully into account based on vehicle dynamics. We established 3 DOF vehicle dynamics model included lateral speed, sideslip and yaw angle, obtained vehicle steering stability equations, and performed simulation experiments to verify the model is valid and reasonable.
\end{abstract}

\section{Introduction}

Vehicle motion simulator is booming as the development of computer simulation technology, aerospace technology and virtual reality technology, which is representative in simulation technology due to its fast growing and wide application [1]. It is important to establish the correct kinetic model in vehicle motion simulator. A more precise and complexity model will costs more computer space. In this paper, we made a compromise between accuracy and complexity of the dynamic model, and established of a of 3 DOF vehicle dynamics model including lateral speed, roll angle and yaw angle.

\section{The establishment of vehicle steering stability model}

In driving simulation applications, the driver have to face an issue of veer, so it is essential to establish an accurate vehicle steering dynamic equation [5].
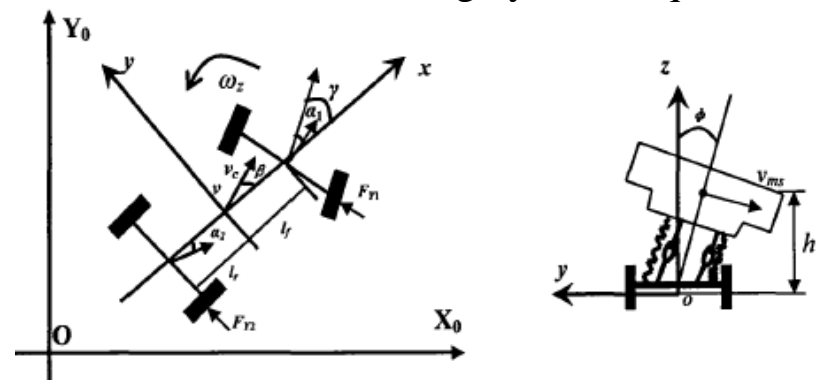

Fig 2-1 force situation of the vehicle in veer

The vehicle mass is divided in two parts, the top of suspension and the rest, and the former will have a roll motion in veer. The car steering force in veer is shown in Figure 2-1. Coordinate system(OX0Y0Z0) is absolute coordinate system which is fixed to the ground, and coordinate system (OXYZ) is vehicle reference coordinate system. The point $\mathrm{O}$ coincides with the center of non-suspension mass. When the vehicle front wheels turned an angle of $\gamma$, a centrifugal force generated by the center of mass caused the lateral reaction forces $F_{Y 1}$ and $F_{Y 2}$ on the front and rear wheels respectively which produced sideslip angle of $\alpha_{1 \text { and }} \alpha_{2}$. The yaw velocity is $w_{z}$. Angle $\beta$ is from the car x-axis to centroid velocity., $l_{f}, l_{r}$ stand for the distance of the centroid to the former

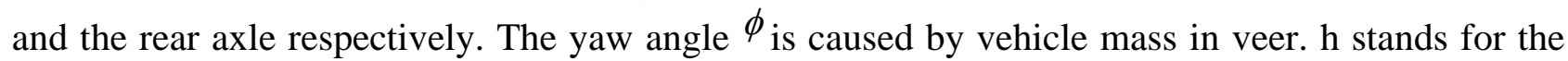
height difference between the suspension centroid to the vehicle's roll center.

Vehicle steering force satisfies equilibrium conditions in axils, so there are three force equilibrium equations and three torque equilibrium equations [6]. It is assumed that there are not pitching and vertical movement, sliding phenomenon in veer, aligning torque, the impact of the suspension and steering system, and tire characteristics changes caused by load changes. Front 
wheel steering angle $\gamma$ is regard as input. In this case, the model will be simplified to 3-DOF, yaw

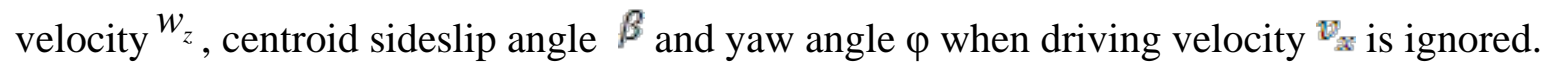

Because the movement of $\mathrm{z}$-axis direction perpendicular is ignored, the velocity of nonsuspension centroid $\mathrm{O}$ in vehicle coordinate system is given by equation (2-1),

$$
\vec{v}_{c}=u \vec{i}+v \vec{j}
$$

There is only a yaw rotation transformation which is about z-axis between vehicle coordinate system and ground absolute coordinate system, so it is given by equation (2-2) in terms of yaw angle $\varphi$, and then the velocity $\overrightarrow{v_{e}}$ of point $\mathrm{O}$ in ground coordinate system is given by equation (2-3).

$$
\begin{aligned}
&\left\{\begin{array}{l}
X=r \cos \Delta \\
Y=r \sin \Delta \\
x=r \cos (\Delta-\varphi) \\
y=r \sin (\Delta-\varphi)
\end{array} \Rightarrow\left[\begin{array}{l}
x \\
y
\end{array}\right]=\left[\begin{array}{ll}
\cos \varphi & \sin \varphi \\
-\sin \varphi \cos \varphi
\end{array}\right]\left[\begin{array}{l}
X \\
Y
\end{array}\right]\right. \\
& \vec{v}_{e}=(u \cos \varphi-v \sin \varphi) \vec{x}+(u \sin \varphi+v \cos \varphi) \vec{y}
\end{aligned}
$$

The acceleration $\overrightarrow{a_{0}}$ can be acquired by deviation of $\overrightarrow{v_{e}}$,

$$
\begin{aligned}
\vec{a}_{0} & =(\dot{v} \cos \varphi-\mathrm{u} \dot{\varphi} \sin \varphi-\dot{v} \sin \varphi-v \dot{\varphi} \cos \varphi) \vec{x}+(\dot{v} \sin \varphi-\mathrm{u} \dot{\varphi} \cos \varphi+\dot{v} \sin \varphi \\
& -v \dot{\varphi} \sin \varphi) \overrightarrow{\mathrm{y}}
\end{aligned}
$$

The acceleration $\overrightarrow{a_{0}}$ can be expressed in vehicle coordinate system due to the equation $\varphi=\omega_{z}$

$$
\vec{a}_{0}=\left[\begin{array}{l}
a_{x} \\
a_{y}
\end{array}\right]=\left[\begin{array}{cc}
\cos \varphi & \sin \varphi \\
-\sin \varphi & \cos \varphi
\end{array}\right]\left[\begin{array}{l}
a_{x} \\
a_{y}
\end{array}\right]=\left[\begin{array}{c}
\dot{v}-v \omega_{z} \\
\dot{v}+u \omega_{z}
\end{array}\right]
$$

The angle $\beta$ between the vehicle centroid velocity $v_{c}$ and $x$-axis is small as shown in figure 2-1, so the vehicle forward velocity $\mathrm{u}$ and velocity $\mathrm{v}$ along the $\mathrm{y}$-axis direction are given by equation (2$6)$,

$$
\left\{\begin{array}{l}
u=v_{c} \cos \beta \approx v_{c} \\
v=v_{c} \sin \beta \approx u \beta
\end{array}\right.
$$

It is assumed that the forward velocity $\mathrm{u}$ is a constant in veer, so the velocity of the centroid $\mathrm{O}$ is given by equation (2-7),

$$
\vec{a}_{0}=\left(\mathrm{u} \beta \omega_{z}\right) \overrightarrow{\mathrm{i}}+u\left(\dot{\beta}+\omega_{z}\right) \overrightarrow{\mathrm{j}}
$$

The velocity $\overrightarrow{v_{M O}}$ and acceleration $\overrightarrow{a_{M O}}$ of M-point relative O-point are given by equation (2-8) and (2-9) according to the position vector $\mathrm{R}$ and angle velocity $\Omega$,

$$
\begin{aligned}
& \vec{v}_{M o}=\Omega \times R=-\omega_{z} y \vec{i}+\left(\omega_{z} x-\omega_{x} z\right) \vec{j}+\omega_{x} y \vec{k} \\
& \vec{a}_{M o}=\frac{d \vec{v}_{M o}}{d t}
\end{aligned}
$$

According to equation (2-7) (2-9), the acceleration of any point $\mathrm{M}$ in the vehicle suspension can be given by equation (2-10),

$$
\begin{aligned}
\vec{a}_{M}= & \vec{a}_{o}+\vec{a}_{M 0}=\left(-u \beta \omega_{z}-\omega^{2} x-\dot{\omega}_{z} y+\omega_{x} \omega_{z} z\right) \vec{i}+\left[u\left(\dot{\beta}+\omega_{z}\right)+\dot{\omega}_{z} x-\right. \\
& \left.\left(\omega_{x}^{2}+\omega_{z}^{2}\right) y-\dot{\omega}_{x} z\right) \vec{j}+\left(\omega_{x} \omega_{z} x-\dot{\omega}_{x} y-\omega_{x}^{2} z\right) \vec{k}
\end{aligned}
$$

Force and moment equilibrium equation can be acquired using Alembert's principle, 


$$
\left\{\begin{array}{c}
m u\left(\dot{\beta}+\omega_{z}\right)-\mathrm{m}_{s} h \dot{\omega}_{x}=2 k_{1} \gamma-2\left(k_{1}+k_{2}\right) \beta-2\left(k_{1} l_{f}-k_{2} l_{r}\right) \frac{\omega_{z}}{u} \\
I_{z} \dot{\omega}_{z}+I_{x z} \dot{\omega}_{x}=F_{Y 1} l_{f}+F_{Y 2} l_{r}=2 k_{1} \alpha_{1} l_{f}+2 k_{2} \alpha_{2} l_{r} \\
I_{x} \dot{\omega}_{x}+I_{x z} \dot{\omega}_{z}-\mathrm{m}_{s} h u\left(\dot{\beta}+\omega_{z}\right)=\mathrm{L}_{\phi} \phi+\mathrm{L}_{\omega} \omega_{x}
\end{array}\right.
$$

Where, $k_{1}, k_{2}$ stands for the front and rear tire side stiffness respectively, and $\alpha_{1}, \alpha_{2}$ is sideslip angle. $L_{\varphi}$ is an external torque generated by unit yaw angle because of suspension elasticity and gravity $L_{w}$ is an external torque generated by unit yaw angle because of damper.

So far, the vehicle 3-DOF steering balance dynamics equations are all given by equation (2-1) during the car is veering. Algebraic iteration equations can be abstained by solving state-variable $\left\{\beta, \omega_{z}, \phi, \omega_{x}\right\}$ at any time using numerical methods in a known initial state.

\section{Solving differential equations of vehicle dynamics model}

Vehicle steering stability equations belong to differential equations with constant coefficients, which can be solved by Euler method, improved Euler method, Adams pre-estimated method and Runge-Kutta method [7].Considering the accuracy and computing speed requirements, we use fourth-order Runge-Kutta method. The differential equations in the previous section can be written as:

$$
\left\{\begin{array}{l}
a_{1} \beta+a_{2} \dot{\beta}+a_{3} \omega_{z}+a_{4} \dot{\omega}_{z}+a_{5} \phi+a_{6} \dot{\phi}+a_{7} \ddot{\phi}=d_{1} \\
b_{1} \beta+b_{2} \dot{\beta}+b_{3} \omega_{z}+b_{4} \dot{\omega}_{z}+b_{5} \phi+b_{6} \dot{\phi}+b_{7} \ddot{\phi}=d_{2} \\
c_{1} \beta+c_{2} \dot{\beta}+c_{3} \omega_{z}+c_{4} \dot{\omega}_{z}+c_{5} \phi+c_{6} \dot{\phi}+c_{7} \ddot{\phi}=d_{3}
\end{array}\right.
$$

Where, let $x_{1}=\beta_{3} x_{2}=\omega_{z}, x_{3}=\phi_{3} x_{4}=\dot{\phi}$, and the matrix is expressed as

It is simplified into equation (3-3),

$$
\left[\begin{array}{cccc}
a_{2} & a_{4} & 0 & a_{7} \\
b_{2} & b_{4} & 0 & b_{7} \\
c_{2} & c_{4} & 0 & b_{7} \\
0 & 0 & 1 & 0
\end{array}\right]\left[\begin{array}{l}
\dot{a}_{1} \\
\dot{a}_{2} \\
\dot{a}_{3} \\
\dot{a}_{4}
\end{array}\right]=\left[\begin{array}{cccc}
-a_{1} & -a_{3} & -a_{5} & -a_{6} \\
-b_{1} & -b_{3} & -b_{5} & b_{6} \\
-c_{1} & -c_{3} & -c_{5} & c_{6} \\
0 & 0 & 0 & 1
\end{array}\right]\left[\begin{array}{c}
x_{1} \\
x_{2} \\
x_{3} \\
x_{4}
\end{array}\right]+\left[\begin{array}{c}
d_{1} \\
d_{2} \\
d_{3} \\
0
\end{array}\right]
$$

For the first-order linear differential equations,

$$
\dot{x}=A^{-1} B x+A^{-1} b=C^{-1} x+d
$$

$$
\left\{\begin{array}{l}
\frac{d x_{1}}{d t}=f\left(t, x_{1}, x_{2}, \ldots x_{n}\right) \\
\frac{d x_{2}}{d t}=f\left(t, x_{1}, x_{2}, \ldots x_{n}\right) \\
\cdots \\
\frac{d x_{n}}{d t}=f\left(t, x_{1}, x_{2}, \ldots x_{n}\right)
\end{array}\right.
$$

The iterative formula with fourth-order Runge-Kutta method by integral from $t_{j}$ to $t_{j+1}=t_{j}+h$ is as follows,

$$
x_{i, j+1}=x_{i j}+\frac{h}{6}\left(K_{1 i}+2 K_{2 i}+2 K_{3 i}+K_{4 i}\right)
$$

Where, 
The simulation analysis of vehicle dynamics model

$$
\left\{\begin{array}{l}
K_{1}=f_{i}\left(t_{j}, x_{1 j}, x_{2 j}, \ldots x_{n j}\right) \\
K_{2}=f_{i}\left(t_{j}+\frac{h}{2}, x_{1 j}+\frac{h}{2} K_{11}, \ldots x_{n j}+\frac{h}{2} K_{1 n}\right) \\
K_{3}=f_{i}\left(t_{j}+\frac{h}{2}, x_{1 j}+\frac{h}{2} K_{21}, \ldots x_{n j}+\frac{h}{2} K_{2 n}\right) \\
K_{4}=f_{i}\left(t_{j}+h, x_{1 j}+h K_{31}, \ldots x_{n j}+h K_{3 n}\right)
\end{array}\right.
$$

In order to verify the correctness of vehicle dynamics model, we made a data comparison between experimentation and simulation in two typical road conditions, and let step size $\mathrm{h}=0.02 \mathrm{in}$ equation (3-6).

(1)The condition of turning right at a low speed

Experimental vehicle turned right around $90^{\circ}$ at a low speed $(10 \mathrm{~km} / \mathrm{h})$ and continued driving. There are a set of comparisons between parametric curves generated by simulation of dynamics model and road test data shown in Figure 4-1 4-4.

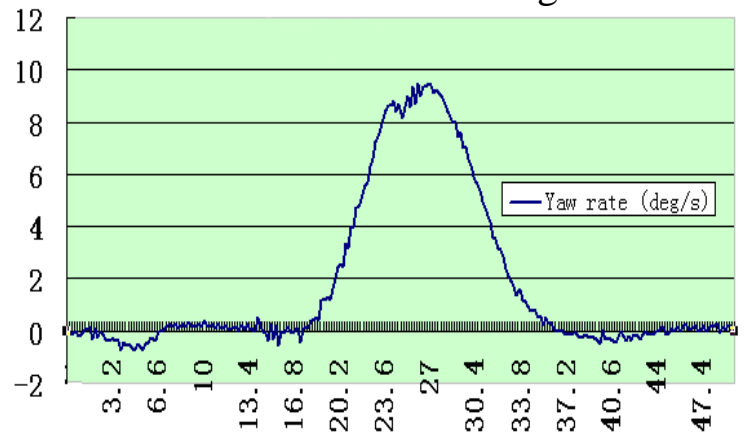

Fig 4-1 Yaw rate curve of road test

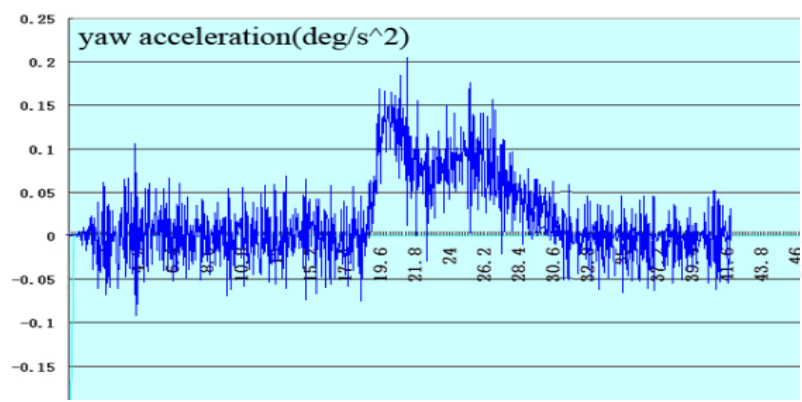

Fig 4-3 Yaw acceleration of road test

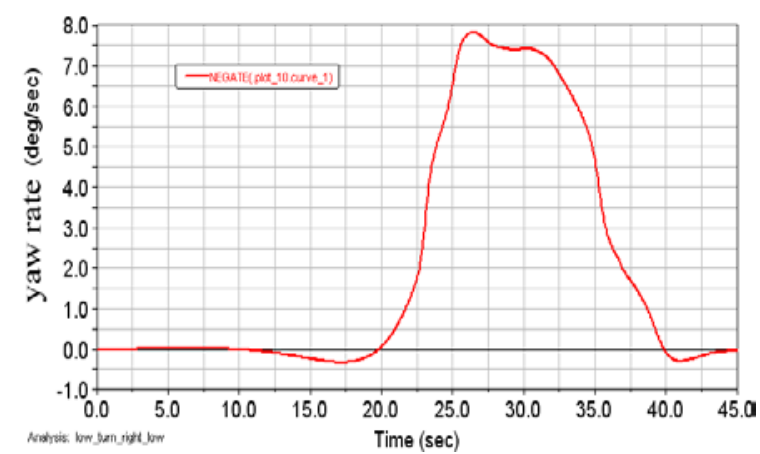

Fig 4-2 Yaw rate curve of simulation

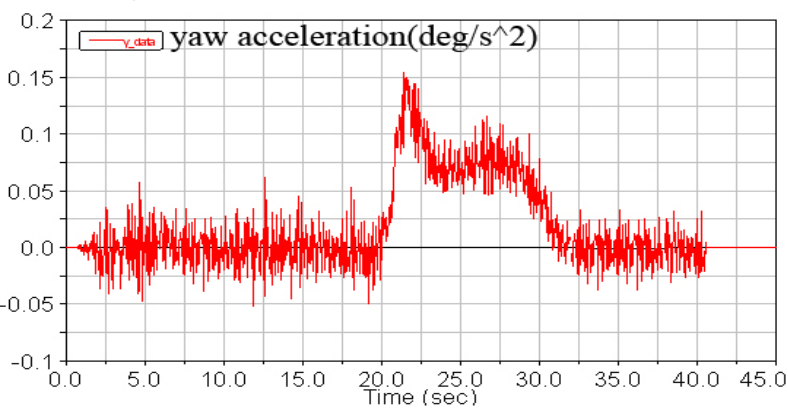

Fig 4-4 Yaw acceleration of simulation

(2) The condition of changing lane

During the straight driving at a constant velocity $(40 \mathrm{~km} / \mathrm{h})$, experimental vehicle changed lane in front of barrier, and remained constant speed. There are a set of comparisons between simulation and road test data shown in Figure 4-5 4-8.

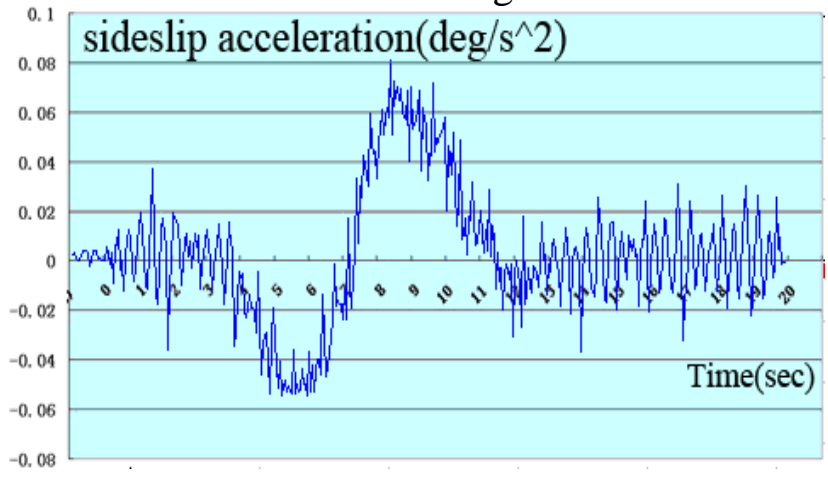

Fig 4-5 Sideslip acceleration of road test

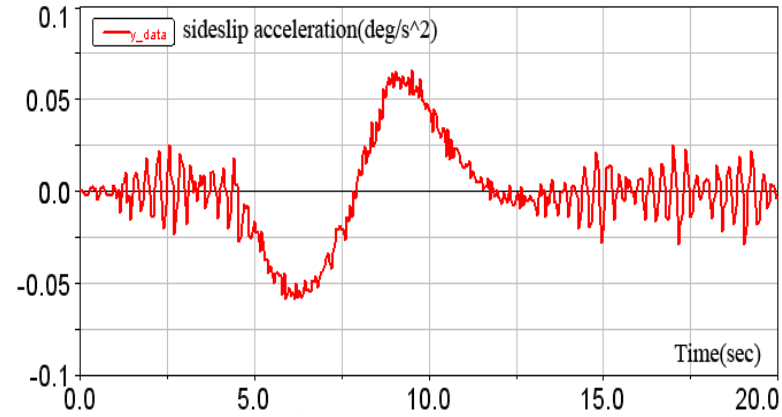

Fig 4-6 Sideslip acceleration of simulation 


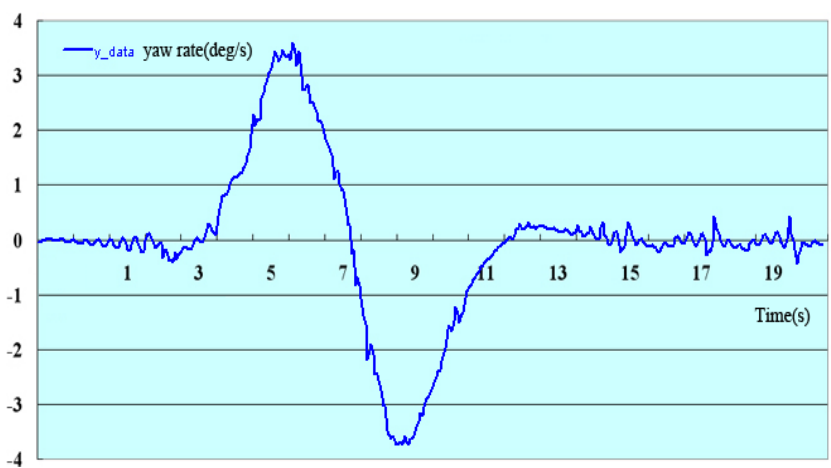

Fig 4-7 Yaw rate curve of road test

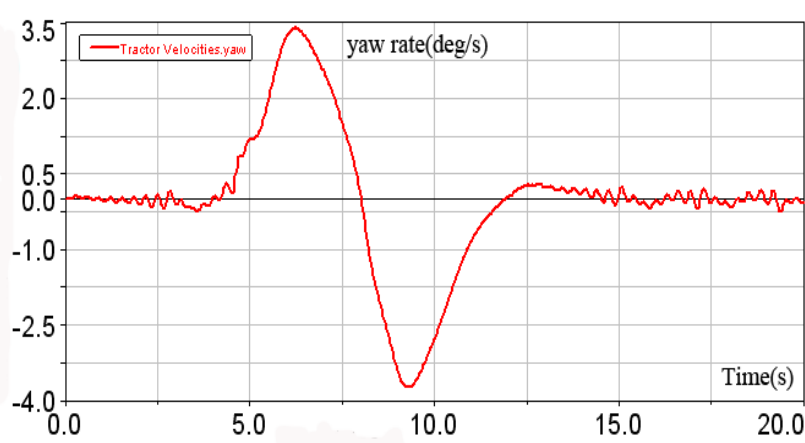

Fig 4-8 Yaw rate curve of simulation

As can be seen from the comparisons of the two conditions, there are a highly similar curve and small numerical errors between simulation results and real road test data. Because of some randomness of driver and road in real test operation, there are some fluctuations in the experimental result, but the simulation results are still very close to the actual test.

\section{Conclusions}

It can be concluded that the 3-DOF vehicle dynamics model was reasonable and correct which contained lateral speed, sideslip and yaw angle. It is shown that there are a highly similar curve and small numerical errors in simulation results. The vehicle dynamics model with high accuracy was verified by road test, and it accurately reflected the true state of vehicle performance.

\section{References}

[1] Lapiska C. Flight simulation: Overview. Aerospace America. 1993, (8): 12-16.

[2] David H.Eberly. 3D Game Engine Design: A Practical Approach to Real-Time Computer Graphics, Morgan Kaufmann, 2000.

[3] M.Mischke . Automobile Dynamics. Beijing: China Machine Press. 1980.

[4] Wang Wei, Wu Chaozhong, Yan Xinping, etc. Dynamics Simulation Modeling of Vehicle Driving Simulator. Wuhan Technology University Journal. 2005,2.

[5] Guo Konghui. Vehicle Handling Dynamics. Changchun: Jilin Science and Technology Press. 1991.

[6] Lei Yucheng. Vehicle System Dynamics and Simulation. Beijing: National Defense Industry Press. 1997.

[7] Steven C. Chapra. Numerical Methods in Engineering. Beijing: Science Press. 2000. 